\title{
Thermal Properties of Concrete Incorporated with Shape-stable Phase Change Material
}

\author{
Chun On Chin ${ }^{1}$ and Ying Kong Sih ${ }^{1, *}$ \\ ${ }^{1}$ Discipline of Civil Engineering, School of Engineering, Monash University Malaysia, Malaysia
}

\begin{abstract}
There has been an ever-increasing interest in concrete incorporated with shape-stable phase change material (SSPCM) in recent years for its outstanding thermal performance. In this research, PCM was incorporated into porous lightweight aggregate, namely exfoliated vermiculite to form SSPCM. SSPCMS were integrated with concrete to study their effects on thermal behaviour. Thermal testing was performed using both hot plate and KD2Pro. From the obtained results, it was observed that thermal conductivity and diffusivity reduced by $29 \%$ and $63 \%$ respectively whereas specific heat capacity increased by $40 \%$ with inclusion of SSPCMs. It was concluded that the implementation of SSPCM technology can be seen as a feasible and economical solution for energy efficient buildings.
\end{abstract}

\section{Introduction}

Due to the growth of population, the energy demand for buildings has been increasing and it is forecasted that the demand will continue to increase rapidly in coming years. From the past data, the building sector contributes around $27 \%$ of overall energy consumption and it is predicted to be one of the highest carbon dioxide emitter by 2035 (Vicente and Silva, 2014). Hence, the development of sustainable buildings has attracted great amount of attention from researchers to enhance energy efficiency of buildings.

Phase change material (PCM) is an excellent candidate for energy storage medium as it can absorb heat when there is a surplus of heat energy and then releases energy when the ambient temperature drops (Kalnaes and Jelle, 2015). Besides that, PCM is able to store 514 times more heat compared to typical construction materials such as masonry and rock (Karim et al., 2014).

There are several methods to incorporate PCM into buildings materials including direct incorporation and immersion, form-stable composite PCM and encapsulation technique. The first method is the easiest to carry out but leakage of the PCM has been reported (Soares et al., 2013). The leakage of PCM will greatly affect the thermal performance and structural stability of the concrete. The leakage issue can be rectified by the form-stable composite PCM and encapsulation technique, hence successfully grabbed the attention of many researchers in the past decade.

By incorporating PCM into porous aggregates, shape-stable phase change materials (SSPCM) can be formed. The integration of SSPCM into concrete is able to enhance the

* Corresponding author: kong.sih.ying@monash.edu 
thermal mass of buildings and reduces the indoor temperature at the same time. By employing this technique, the PCM is contained inside of porous aggregates due to the surface tension and capillary forces developed between the PCM and porous aggregates. Hence, the stability of PCM is dependent on the porosity of the materials (Cabeza et al., 2007).

SSPCM possess a high enthalpy of fusion which is important to improve the thermal inertia of buildings. The higher the enthalpy of fusion, the longer the temperature can be preserved within the preferred range (Kalnaes and Jelle, 2015).

Nevertheless, the absence of encapsulation on SSPCM could result in leakage of PCM. Mehrali et al. (2014), concluded that the leakage of PCM would occur when the surrounding temperature is greater than their melting points. Leakage is one of the serious issue that affects the physical properties of the concrete and also the thermal performance of the PCMs which causes the instability in concrete structure.

Memon et al. (2015), carried out a research using porous lightweight aggregates to retain PCM. The PCM was impregnated using vacuum impregnation method and then sealed by using epoxy to prevent leakage of PCM. It is proved that different coating methods might result in different impact on thermal properties of SSPCM such as lower thermal conductivity which would likely affect the thermal performance of concrete. In order to resolve the leakage issue and provide SSPCM with satisfactory thermal properties, graphite powder could be included in the epoxy to enhance the thermal conductivity of the coating.

\section{Methodology}

\subsection{Selection of Materials}

The selected PCM was an organic paraffin (RT31) with molecular formula $\mathrm{CnH} 2 \mathrm{n}+2$ of saturated hydrocarbons manufactured by Rubitherm ${ }^{\circledR}$. The paraffin was chosen due to its desirable properties such as non-corrosive, does not suffer from super-cooling, and high chemical stability during the cycles of phase change process. Furthermore, its phase change temperature is within the human comfort zone and it has decent latent heat storage capacity. Specifications of paraffin used including melting point started at $29^{\circ} \mathrm{C}$, thermal conductivity of $0.2 \mathrm{~W} / \mathrm{mK}$, specific heat of $2 \mathrm{~J} / \mathrm{kgK}$, and heat capacity of $165 \mathrm{~kJ} / \mathrm{kg}$.

Medium sized lightweight aggregate (LWA), exfoliated vermiculite was chosen as the carrier for paraffin. It has high absorption capacity, which grants it the capability to retain paraffin within its inter-laminar voids. Generally, the bulk density of exfoliated vermiculite is 10-12 times less than the crude vermiculite. The particle size of exfoliated vermiculite ranging from $1.0-2.5 \mathrm{~mm}$ with a bulk density of $150 \mathrm{~kg} / \mathrm{m}^{3}$.

In order to resolve the leakage problem, Aerosil R202 from Evonik ${ }^{\circledR}$ was used as a coating agent. It is a hydrophobic material with a specific surface are of $80-100 \mathrm{~m}^{2} / \mathrm{g}$ and an average particle size of $14 \mathrm{~nm}$ which can be used to produce the surface tension between paraffin and LWA.

\subsection{Preparation of SSPCMs Composite and SSPCM Concrete Panel}

The moisture content of exfoliated vermiculite was first removed by heating in an oven at a temperature of $105^{\circ} \mathrm{C}$ over 24 hours. The mass of samples before and after drying were recorded. RT31 was placed in the oven at $45^{\circ} \mathrm{C}$ until it became liquid form. After the 
paraffin was fully melted, it was placed in a mixing vessel and the dried vermiculite was gradually added into the paraffin.

After the immersion process, Aerosil R202 was applied to the PCM composite. It was believed that the leakage issue can be prevented by the coating agent deposited on the surface of the aggregates. This system is also known as dry powder treatment as Aerosil played the role of host of dried particles on the SSPCM composites.

Eight mix designs were considered as shown in Table 1 in order to evaluate the thermal properties of the concrete incorporated with SSPCM. The mixing process involved several steps. Firstly, the cement and sand were to mix thoroughly for about 2 minutes. Secondly, the water was added gradually and the mixing continued for another two minutes. Lastly, the SSPCM composite was added into the mixture and mixed for two minutes. It is important to note that the SSPCM composite shall be added last to minimize damage of the encapsulation of the SSPCM due to the high shear force involved in the mixing process.

Table 1. Mix proportion of the samples

\begin{tabular}{|c|c|c|c|c|c|}
\hline Panel & Mix Design & Ratio & Panel & Mix Design & Ratio \\
\hline S1 & C:S & $1: 2$ & S5 & C:S:V & $1: 2.85: 0.15$ \\
\hline S2 & C:S & $1: 4$ & S6 & C:S:V & $1: 2.55: 0.45$ \\
\hline S3 & C:S:A & $1: 2: 4$ & S7 & $\begin{array}{c}\text { C:S:V (with 5\% } \\
\text { of RT31) }\end{array}$ & $1: 2.85: 0.15$ \\
\hline S4 & C:S:A & $1: 1.3: 1.3$ & S8 & $\begin{array}{c}\text { C:S:V (with } 8 \% \\
\text { of RT31) }\end{array}$ & $1: 2.85: 0.15$ \\
\hline
\end{tabular}

${ }^{*} \mathrm{C}$ is cement, $\mathrm{S}$ is Sand, $\mathrm{A}$ is aggregate and $\mathrm{V}$ is Vermiculite

\subsection{Thermal Properties Testing using hot plate and KD2Pro}

Two concrete panels with a dimension of $200 \times 200 \times 50 \mathrm{~mm}$ were fabricated for each mix design. In order to determine the thermal properties of the concrete panels, a simple thermal testing was assembled using a hot plate, thermocouples and a data logger. The accuracy of the thermocouples and hot plate was $\pm 0.2 \%$ and $\pm 0.5 \%$ respectively.

The concrete panel was placed on the hot plate and five thermocouples were placed on top of the concrete panel to measure the temperature with respect to time and the temperature time histories were recorded using a data logger. The thermocouples were placed at the corners and centre of the concrete panel to ensure consistency of the temperature readings. Each of the concrete panels was tested using the hot plate at a temperature of $35^{\circ} \mathrm{C}$ and $45^{\circ} \mathrm{C}$ for a period of 8 hours.

KD2Pro is a device that measures thermal properties in compliance with ASTM D533414. This device is able to measure thermal properties including thermal conductivity, thermal resistivity, thermal diffusivity, and specific heat within the accuracy of $\pm 10 \%$. Before conducting thermal testing using KD2Pro, performance verification and calibration must first be done. The surrounding temperature of the system will greatly affect the accuracy of measurement; hence the temperature of the surroundings has to be relatively constant before the measurement started.

The measurement was taken by inserting the probe fully in the concrete panel. The probe consists of a needle with a heater and sensor within it which is able to simulate extremely long and thin thermal source. A current is passed through the heater and analysis of the sensor temperature can determine the thermal conductivity during the heating and cooling. All the information will be recorded in the data logger. 


\section{Results and Discussion}

Figures $1 \& 2$ illustrate the responses of samples when subjected to an applied heat source at different temperatures. It was noted that at the beginning of the heating process, the rate of temperature increment was similar for all the samples. After certain period of heating, the rate of temperature increment for samples incorporated with SSPCM began to decrease when compared to normal lightweight concrete. This indicated that the melting of paraffin delayed the heat energy from reaching the cold side of the panels. In other words, the surrounding temperature was absorbed during the phase change process of paraffin.

Lightweight concrete using vermiculite is typically recognized as a good heat insulator. However, when analysing the plateau temperature, it did not reduce the plateau temperature significantly compared to other normal concrete and mortar under the temperature of $35^{\circ} \mathrm{C}$. It showed a $1.63^{\circ} \mathrm{C}$ to $2.32^{\circ} \mathrm{C}$ reduction in plateau temperature when applied to the temperature of $45^{\circ} \mathrm{C}$. Hence, it could be concluded that exfoliated vermiculite was only able to show its thermal insulator behaviour at a higher temperature.

Phase change material such as paraffin is also well known as a good heat insulator due to its low thermal conductivity. From the results, it showed that paraffin was able to reduce the temperature of the top surface of concrete panels. With addition of $5 \%$ of paraffin, the final temperature was reduced by $1.06{ }^{\circ} \mathrm{C}$ and $1.59{ }^{\circ} \mathrm{C}$ under the temperature of $35^{\circ} \mathrm{C}$ and $45{ }^{\circ} \mathrm{C}$ respectively. On the other hand, with addition of $8 \%$ of paraffin, the final temperature was reduced by $1.63{ }^{\circ} \mathrm{C}$ and $1.91{ }^{\circ} \mathrm{C}$ under the temperature of $35^{\circ} \mathrm{C}$ and $45^{\circ} \mathrm{C}$ respectively. This was attributed to the high latent heat of paraffin which allowed storing of heat energy transferred to the panels.

As for the time for the concrete panels to reach temperature plateau, it is an important parameter to measure the heat capacity. The longer the duration, the higher the specific heat capacity. It was obvious that both exfoliated vermiculite and paraffin showed an increment in duration hence the specific heat capacity was also expected to be higher. This implied that the higher the content of paraffin, the greater amount of energy was required to raise the temperature.

The results also indicated that with greater amount of heat applied, the concrete panels could reach the temperature plateau quicker. This scenario become more distinct with SSPCM composite. This was mainly due to the particles within the concrete panels vibrated more rapidly when it received greater heat energy and thus allowed the increase of temperature in shorter period. 


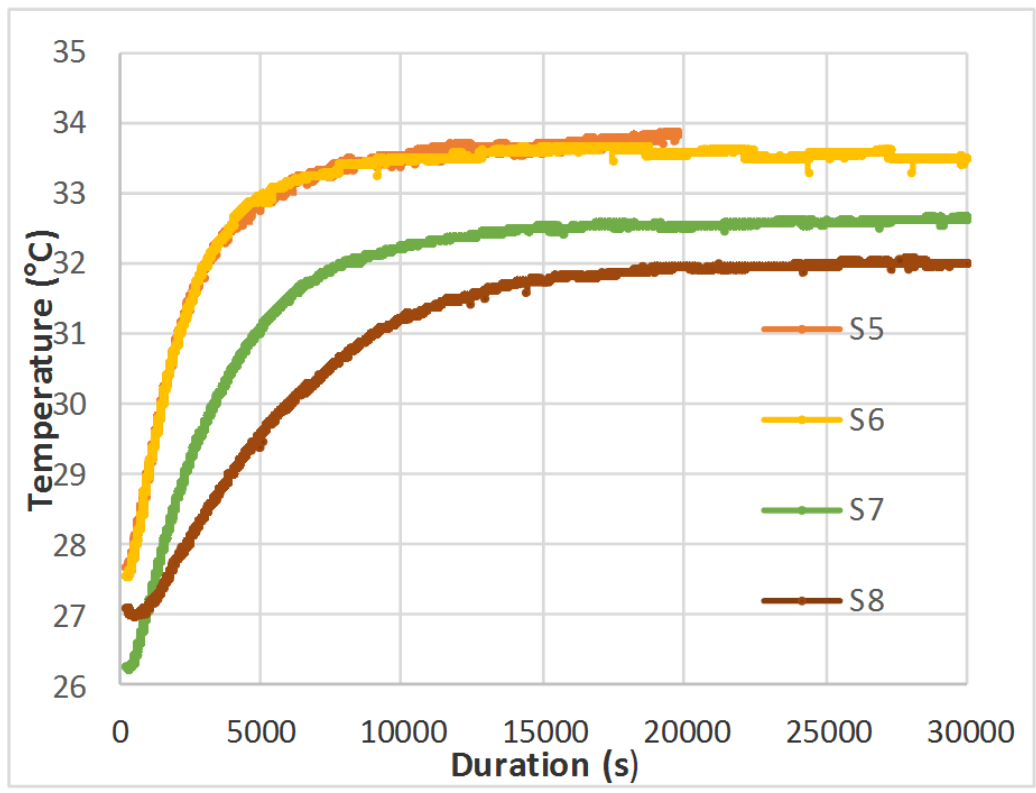

Fig. 1. Temperature time histories of concrete panels subjected to $35^{\circ} \mathrm{C}$

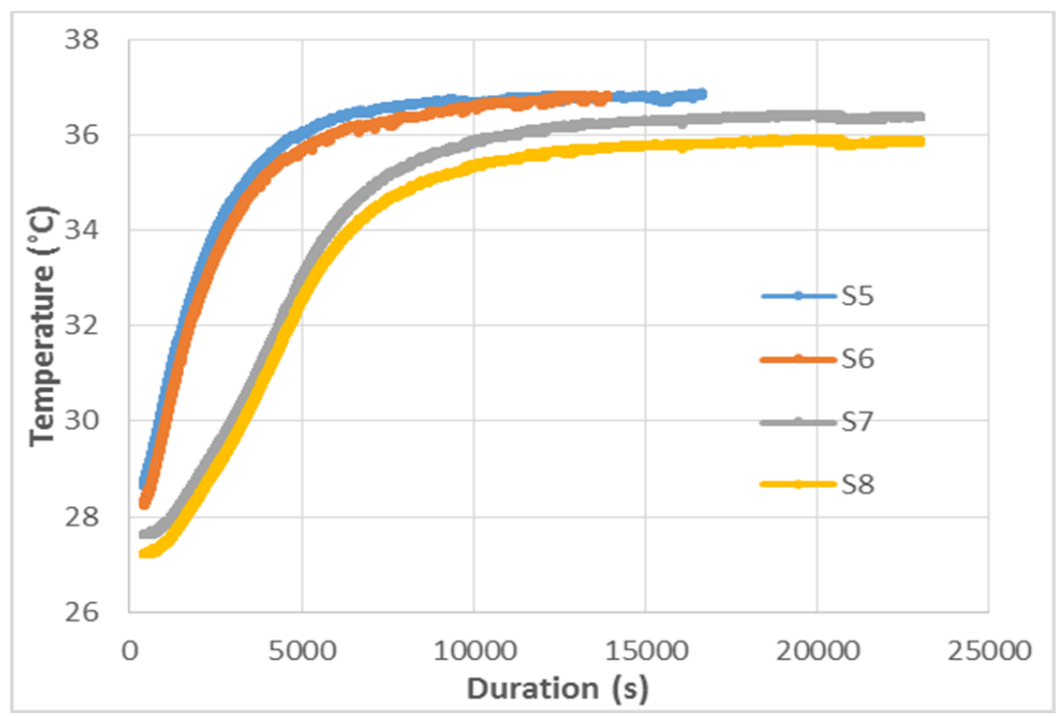

Fig. 2. Temperature time histories of concrete panels subjected to $45^{\circ} \mathrm{C}$

\subsection{Thermal Conductivity}

The thermal conductivity of different panels using various experimental methods is summarized in Table 2 and Table 3. Both hot plate and KD2Pro yielded similar results. Therefore, it was evident that by integrating SSPCMs, the thermal conductivity would be decreased significantly. The lower thermal conductivity reduced the capability of transmitting heat through the panel, which developed once the PCM has fully melted. The melted PCM will release heat when a lower temperature is imposed. This is of great 
importance as it enhances longer response from the SSPCM to the thermal condition applied.

With addition of 5\% RT31 paraffin, the thermal conductivity dropped by $18 \%$ to $22 \%$. While with addition of $8 \%$ of paraffin, the thermal conductivity dropped by $25 \%$ to $29 \%$. This provided a large buffer zone which restricted the transfer of heat successfully. These results proved that SSPCM is applicable in real industry due to its viability in restricting the heat transfer while having minimal effect on the physical properties of concrete. However, there are times when the ability to transmit heat is actually preferred. Higher thermal conductivity is needed when SSPCM functions to release the inner heat of the building during the night time.

Table 2. Summary table of thermal conductivity using different device

\begin{tabular}{|c|c|c|c|c|c|c|c|c|}
\hline $\begin{array}{c}\text { Thermal } \\
\text { Conductivity } \\
\text { (W/mK) by } \\
\text { Different Device }\end{array}$ & S1 & S2 & S3 & S4 & S5 & S6 & S7 & S8 \\
\hline $\begin{array}{c}\text { using hot plate } \\
\text { at } 35^{\circ} \mathrm{C}\end{array}$ & 1.924 & 1.336 & 1.374 & 1.761 & 1.503 & 1.230 & 1.234 & 0.894 \\
\hline $\begin{array}{c}\text { using hot plate } \\
\text { at } 45^{\circ} \mathrm{C}\end{array}$ & 2.016 & 1.934 & 1.882 & 2.298 & 1.496 & 1.556 & 1.229 & 1.172 \\
\hline (using KD2Pro) & 2.218 & 2.598 & 2.613 & 2.483 & 1.608 & 1.409 & 1.259 & 1.003 \\
\hline
\end{tabular}

Table 3. Percentage difference in thermal conductivity using different device

\begin{tabular}{|c|c|c|}
\hline & $\begin{array}{c}\text { \% difference in thermal } \\
\text { conductivity between S5\&S7 }\end{array}$ & $\begin{array}{c}\text { \% difference in thermal } \\
\text { conductivity between S6\&S8 }\end{array}$ \\
\hline using hot plate at $35{ }^{\circ} \mathrm{C}$ & $18 \%$ & $27 \%$ \\
\hline using hot plate at $45{ }^{\circ} \mathrm{C}$ & $18 \%$ & $25 \%$ \\
\hline (using KD2Pro) & $22 \%$ & $29 \%$ \\
\hline
\end{tabular}

\subsection{Thermal Diffusivity}

In heat transfer analysis, thermal diffusivity describes the rate of heat transfer from a side with higher temperature to another side. In other word, thermal diffusivity also represents the measure of thermal inertia which is related to thermal lag. Thermal lag is highly dependent on both heat resistivity and heat storage that can reduces the amplitude of the heat transfer.

The thermal diffusivity of different mix samples is tabulated in Table 4. It was observed that with incorporation of SSPCMs, the thermal diffusivity decreased by approximately $63 \%$. This was mainly due to the contribution of latent heat of PCM when raising the temperature near to its melting point, hence increasing the effective heat capacity of panels, which induced a thermal lag.

Table 4. Thermal diffusivity of samples

\begin{tabular}{|c|c|c|c|}
\hline Panel & $\begin{array}{c}\text { Thermal Diffusivity } \\
\left(\mathrm{mm}^{2} / \mathrm{s}\right)\end{array}$ & Panel & $\begin{array}{c}\text { Thermal Diffusivity } \\
\left(\mathrm{mm}^{2} / \mathrm{s}\right)\end{array}$ \\
\hline S1 & 1.097 & $\mathrm{~S} 5$ & 0.719 \\
\hline S2 & 0.889 & $\mathrm{~S} 6$ & 0.796 \\
\hline S3 & 0.970 & $\mathrm{~S} 7$ & 0.298 \\
\hline S4 & 1.165 & $\mathrm{~S} 8$ & 0.293 \\
\hline
\end{tabular}




\subsection{Specific Heat Capacity}

Specific heat capacity of different panels was calculated based on the extent of heat flux and the temperature change. From Table 5, it showed that the specific heat capacity increased by $33 \%$ and $40 \%$ with addition of $5 \%$ and $8 \%$ of paraffin respectively. This indicated that the heat was absorbed by the PCM during phase change process, thus increasing the overall effective specific heat capacity of the panels, as more heat energy is required to raise the temperature of the panels. The increment in specific heat capacity also indicated the suitability of incorporating SSPCM into lightweight construction in order to reduce the weight of structures while maintaining its heat capacity.

Table 5. Specific heat capacity of samples

\begin{tabular}{|c|c|c|c|}
\hline Panel & $\begin{array}{c}\text { Specific Heat Capacity } \\
(\mathrm{kJ} / \mathrm{kgK})\end{array}$ & Panel & $\begin{array}{c}\text { Specific Heat Capacity } \\
(\mathrm{kJ} / \mathrm{kgK})\end{array}$ \\
\hline $\mathrm{S} 1$ & 0.827 & $\mathrm{~S} 5$ & 1.361 \\
\hline $\mathrm{S} 2$ & 1.111 & $\mathrm{~S} 6$ & 1.340 \\
\hline $\mathrm{S} 3$ & 1.171 & $\mathrm{~S} 7$ & 2.038 \\
\hline $\mathrm{S} 4$ & 0.852 & $\mathrm{~S} 8$ & 2.239 \\
\hline
\end{tabular}

\section{Conclusion}

The performance of concrete incorporated with SSPCMs was presented in this paper. The major finding according to experimental results could be summarized as below:

-High specific heat capacity and high thermal inertia were observed with addition of SSPCM which can positively influence the thermal comfort within a building structure.

-Lower thermal conductivity and thermal diffusivity were observed with addition of SSPCM which make it a good insulating medium

-Implementation of SSPCM technology in construction is promising but the undesirable effects such as low strength, fire resistance, corrosion, and unknown long-term stability must first be researched upon.

\section{Acknowledgement}

This work was undertaken with funding from Ministry of Higher Education of Malaysia under FRGS grant scheme (Program code. FRGS/1/2015/TK10/MUSM/03/1).

\section{Reference}

L. F. CABEZA, C., CASTELlON, NOGUES, M., MEDRANO, M., LEPPERS, R. \& O. ZUBILLAGA, Use of microencapsulated PCM in concrete walls for energy savings. Energy and Buildings, 39, 113-119(2007).

KALNAES, S. E. \& JELLE, B. P. Phase change materials and products for building applications: A state-of-the-art review and future research opportunities. Energy and Buildings, 94, 150-176(2015). 
KARIM, L., BARBEON, F., GEGOUT, P., BONTEMPS, A. \& ROYON, L. New phasechange material components for thermal management of the light weight envelope of buildings. Energy and Buildings, 68, 703-706(2014).

MEHRALI, M., LATIBARI, S. T., MEHRALI, M., MAHLIA, T. M. I. \& METSELAAR, H. S. C. Effect of carbon nanospheres on shape stabilization and thermal behavior of phase change materials for thermal energy storage. Energy Conversion and Management, 88, 206-213(2014).

MEMON, S. A., CUI, H. Z., LO, T. Y. \& LI, Q. S. Development of structural-functional integrated concrete with macro-encapsulated PCM for thermal energy storage. Applied Energy, 150, 245-257(2015).

SOARES, N., COSTA, J. J., GASPAR, A. R. \& SANTOS, P. Review of passive PCM latent heat thermal energy storage systems towards buildings' energy efficiency. Energy and Buildings, 59, 82-103(2013).

VICENTE, R. \& SILVA, T. Brick masonry walls with PCM macrocapsules: An experimental approach. Applied Thermal Engineering, 67, 24-34(2014). 